\title{
Technique for detecting an electrical connection between the right atrium and right pulmonary venous carina
}

\author{
Ippei Tsuboi ${ }^{1}$, Michio Ogano ${ }^{2}$, Kei Kimura ${ }^{2}$, Hidekazu Kawanaka ${ }^{2}$, Masaharu Tajiri², Jun \\ Tanabe $^{2}$, Tsuyoshi Nohara ${ }^{1}$, Yu-ki Iwasaki ${ }^{3}$, Mitsunori Maruyama ${ }^{1}$, and Wataru Shimizu ${ }^{3}$ \\ ${ }^{1}$ Nihon Ika Daigaku Musashi Kosugi Byoin \\ ${ }^{2}$ Kokuritsu Byoin Kiko Shizuoka Iryo Center Kanja Toshoshitsu Izumi \\ ${ }^{3}$ Nihon Ika Daigaku Fuzoku Byoin
}

December 29, 2021

\begin{abstract}
Introduction: There is increasing evidence of the epicardial connection between the right-sided pulmonary vein (PV) carina and right atrium interrupts right-sided PV isolation after circumferential PV ablation in patients with atrial fibrillation. In such cases, carina ablation is often required. This study aimed to assess the utility of the right atrial posterior wall (RAPW) pacing in the detection of the right-sided epicardial connection (EC), evaluate the requirement for additional carina ablation after circumferential pulmonary vein (PV) ablation depending on the presence of EC, and investigate the clinical characteristics including the amount of epicardial adipose tissue (EAT) in patients with ECs. Methods and Results: Forty-one patients scheduled for PV isolation were enrolled. Before ablation, activation mapping of the LA was prospectively performed during pacing from the RAPW. EC was observed in 12 patients (EC group, 29\%), whereas no EC was observed in the remaining 29 patients (non-EC group, 71\%). For PV isolation, carina ablation was required in addition to circumferential ablation in 7 patients $(58 \%)$ in the EC group, compared to 2 patients $(7 \%)$ in the non-EC group ( $<<0.003)$. Periatrial and intercaval EAT volumes were significantly lower $\left(12.8 \pm 6.2\right.$ vs. $23.1 \pm 13.9 \mathrm{ml} / \mathrm{m}^{2}, \mathrm{p}<0.02$, and $1.1 \pm 0.8 \mathrm{vs} .2 .2 \pm 1.6 \mathrm{ml} / \mathrm{m}^{2}, \mathrm{p}<0.02$, respectively) and the patients were younger $(66.5 \pm 6.6$ vs. $72.4 \pm 8.3$ years, $\mathrm{p}<0.03)$ in the EC group than in the non-EC group. Conclusions: RAPW pacing revealed EC between the RA and right PV carina in nearly a quarter of the patients.
\end{abstract}

\section{Introduction}

Pulmonary vein (PV) isolation is the cornerstone of ablative treatment in patients with symptomatic paroxysmal and persistent atrial fibrillation $(\mathrm{AF}) .{ }^{1}$ It is performed with a wide antral approach is an effective strategy in the prevention of $\mathrm{AF}^{2}$ Wider circumferential lesion areas are associated with longer procedure durations and, occasionally, carina ablation is required to achieve electrical isolation. ${ }^{3}$

Several factors explain the importance of the carina in preventing PV isolation. A postmortem study ${ }^{4}$ demonstrated the transmural myocardial thickness of the venoatrial junction as being the greatest at the carina region. Therefore, a thicker myocardial sleeve may disrupt creation of a transmural lesion. Another postmortem study ${ }^{5}$ described a case with muscular strands connecting the right PVs to the right atrium (RA) at the posterior interatrial groove, which could provide epicardial input from the RA to the right PV carina. Recent clinical studies demonstrated electrophysiological evidence confirming the presence of an epicardial connection (EC) between the PV and the left atrium (LA) or RA in patients with AF who underwent catheter ablation. ${ }^{6-8}$ However, the assessment strategies for EC are not unified (e.g., whether assessment must be performed during sinus rhythm or PV pacing and before or after ablation). Owing to the multiple interatrial connections, including the Bachmann bundle, coronary sinus, and EC between the RA and right $\mathrm{PVs},{ }^{6}$ activation patterns of the LA are assumed to change depending on the origin of the right 
atrial impulses. Therefore, pacing from more than one site may be useful in distinguishing these pathways. Therefore, we hypothesized that an additional RA posterior wall (RAPW) pacing with RA appendage (RAA) pacing can detect ECs of the right pulmonary vein (RPV) carina more clearly in patients with AF undergoing catheter ablation.

Furthermore, factors associated with ECs have not been well clarified. Patients with AF have pathophysiological changes within the atria, such as fibrosis and fatty infiltration, resulting in conduction disturbance..$^{9,10}$ A study using an obese sheep model identified conduction abnormalities related to the infiltration of the posterior left atrial muscle by epicardial fat. ${ }^{11}$ Therefore, we presumed that the amount of epicardial adipose tissue (EAT) can affect the presence of the ECs.

The primary aim of this study was to prove the utility of RAPW pacing compared with RAA pacing in the detection of the ECs. Second, we evaluated the requirement for additional carina ablation after circumferential PV ablation, depending on the LA activation pattern before PV ablation. Third, clinical characteristics including the amount of EAT in patients with ECs were assessed.

\section{Methods}

\section{Study subjects}

The present study was designed as a prospective cross-sectional study and performed in accordance with the tenets of the Declaration of Helsinki. The study protocol was approved by the Institutional Review Board of the Shizuoka Medical Center (approval number: 2019-R32). Written informed consent was obtained from all participants before participation. We recruited 41 patients with highly symptomatic paroxysmal or persistent AF refractory to medical management for circumferential PV isolation between October 2019 and March 2020. The exclusion criteria included previous PV isolation, age $<18$ years, and inability to provide informed consent. Patients who had a history of open-heart surgery were excluded because it could affect ECs. Moreover, patients with an implanted pacemaker were excluded due to difficulties in performing computed tomography image analysis resulting from artifact from the pacemaker leads.

All antiarrhythmic drugs except for amiodarone were discontinued for at least 5 half-lives before the procedure. Amiodarone was discontinued 4 weeks in advance.

\section{Multidetector computed tomography and EAT measurements}

Multislice computed tomography images were acquired from contrast-enhanced electrocardiogram-gated, 80slice multidetector cardiac computed tomography (AquillionPRIME 80; Canon Medical Systems, Tochigi, Japan), before the ablation. For the contrast-enhanced scans, $80 \mathrm{~mL}$ of nonionic contrast media (Omnipaque, Daiichi Sankyo Co., Tokyo, Japan) was injected intravenously at a flow rate of $5 \mathrm{~mL} / \mathrm{s}$, followed by $30 \mathrm{~mL}$ of saline. To obtain adequate gating, patients with a heart rate $>80 /$ min received short-acting beta blockers. The scanning parameters were $80 \mathrm{~mm} \times 0.5 \mathrm{~mm}$ detector collimation, tube voltage of $120 \mathrm{kV}$, gantry rotation time of $350 \mathrm{~ms}$, and $600-800 \mathrm{~mA}$ tube current. Adipose tissue was semi-automatically identified using the threshold attenuation values of -190 to $-40 \mathrm{HU}$ on a Workstation (Ziostation2, Amin, Tokyo, Japan). EAT was defined as the adipose tissue located within the pericardial sac. Periatrial EAT volume was calculated by manual tracing of the sum of the EAT volume of each 0.5 -mm-thick axial slice. We set the bifurcation of the pulmonary artery as the top boundary and the ostium of the coronary sinus as the lower boundary (Figure 1A, B). Furthermore, the EAT of the intercaval area, which indicates the space between the right anteroinferior LA and the posterior RA, was also quantified using the axial slices. The cranial and caudal limits of the intercaval area were between the level of the superior vena cava-RA junction and the bottom of the right inferior PV. The periatrial EAT and intercaval EAT measurements were performed by two independent skilled operators. The inter- and intra-observer correlations for variables measured on computed tomography were 0.982 and 0.996 for periatrial EAT $(\mathrm{P}<0.001)$, and 0.925 and 0.997 for intercaval EAT $(\mathrm{P}<0.001)$, respectively. Periatrial and intercaval EAT volumes were indexed for the body surface area.

\section{Electrophysiological study}


Electrophysiological studies were performed under deep sedation with intravenous propofol, pentazocine, and hydroxyzine hydrochloride. Body surface electrograms and bipolar intracardiac electrograms were monitored continuously and recorded on an EP-WorkMate recording system (Abbott, Saint Paul, MN, USA). A 6Fr Duo-Decapolar catheter (BeeAT, Japan Lifeline Co., Ltd., Tokyo, Japan) was placed into the distal coronary sinus and along the lateral wall of the RA. An intracardiac echocardiography catheter CARTO sound (Biosense Webster, Inc., Diamond Bar, CA, USA) was positioned in the RA to guide transseptal punctures. Two long sheaths (SL0, Agilis, Abbott) were passed into the LA, and LA angiography was performed.

Thereafter, LA electroanatomical mapping was performed during pacing from the distal end of a 10-pole catheter (Snake, Japan Lifeline Co., Ltd.) positioned at two different sites of the RA: (1) the RAA, and (2) the RAPW (posteriorly to the fossa ovalis at the level of the RPV carina as depicted by LA angiography). In the case of AF, electrical cardioversion was performed just before LA mapping. RA pacing was performed at twice the diastolic threshold (2-ms pulse width) at cycle lengths of $750 \mathrm{~ms}$. LA activation maps were acquired only if the conduction time between the RA pacing stimulus and the LA was $>30 \mathrm{~ms}$, to exclude direct far field capture by RA pacing. During the RA pacing, the LA was mapped with a multielectrode mapping catheter (PentaRay; Biosense Webster, Inc.) and the CARTO 3 system CONFIDENSE mapping module with the continuous acquisition of electroanatomical points. Points were accepted only if they passed all the selected filters, including cycle length stability within $40 \mathrm{~ms}$ of the mapped cycle range under RAA or RAPW pacing, local activation time stability with $4 \mathrm{~ms}$, and an electrode position stability within $2 \mathrm{~mm}$. A tissue proximity filter based on catheter location and impedance measurements was used to determine electrode proximity to the cardiac tissue. The steepest negative deflection (-dV/dt) in each distal unipolar signal was used for calculation of the local activation time.

\section{Definition of EC}

We assessed ECs only between the RPV carina and the RA. If the breakthrough site at the RPV carina was present as early as the Bachmann's bundle area (anteroseptal LA), the patients were classified into the EC group during RA pacing. If an EC was observed, it was confirmed that the breakthrough was not anatomically in the closest proximity to the RA and that the local potential of the breakthrough was not dull. If there was no EC, the patients were classified into the non-EC group.

\section{Catheter ablation}

The radiofrequency parameters were set at a temperature of $43{ }^{\circ} \mathrm{C}$ and a power of $40 \mathrm{~W}$ for the anterior LA wall and $30 \mathrm{~W}$ for the posterior LA wall. ${ }^{12}$ If PV isolation was not accomplished by the creation of circumferential lesions at the antral level, cautious remapping to detect gaps with a Pentaray catheter with the CONFIDENSE mapping module was performed. If a gap was identified along the initial encircling lesion, additional radiofrequency application at the same power setting was deployed. If PV isolation was not achieved even after ablation at the antral level, repetitive electroanatomical mapping with the CONFIDENSE mapping module was performed. If the earliest potential was recorded at the carina region, 15-20 W radiofrequency application was performed to the carina while fluoroscopically observing the movement of the right diaphragm to prevent phrenic nerve palsy.

\section{Statistical analysis}

The data were presented as the mean \pm standard deviation (SD) for continuous variables or as number and percentages (\%) for categorical variables. For continuous and categorical variables, differences between the two groups were compared using the Student's $t$-test, Mann-Whitney U test, and Fisher's exact test, respectively. All tests were two sided and a $\mathrm{P}$ value of $<0.05$ was considered statistically significant. All statistical analyses were conducted using SPSS version 21.0 (IBM Corp., Armonk, NY, USA).

\section{Results}

Patient Characteristics 
Twelve (29\%) and $29(71 \%)$ patients were classified into the EC and non-EC groups, respectively (Figure 2). Representative electroanatomical mapping of cases in both groups is shown in Figures 3 and 4 . In all patients in the EC group, breakthroughs were observed during RAPW pacing, not during the RAA pacing. A trend toward shorter conduction time from the RAPW stimulus to the LA and LA excitation time in the EC group was observed compared with the non-EC group $(48.5 \pm 12.2$ vs. $55.6 \pm 14.4, \mathrm{p}=0.18,98.3 \pm 20.2$ vs. $107.2 \pm 19.7, \mathrm{p}=0.43$, respectively); however, the differences were not significant (Table 2 ). The other characteristics of the two groups are described in Table 1. The differences in patient characteristics other than age were nonsignificant (EC-group: $66.5 \pm 6.6$ years vs. non-EC group: $72.4 \pm 8.3$ years, $\mathrm{p}<0.03$ ).

\section{Requirements of carina ablation for PV isolation}

The results of PV isolation by circumferential ablation are shown in Figure 5. PV isolation was achieved by circumferential ablation alone in 5 of the 12 patients (42\%) in the EC group, compared to 27 of 29 patients $(93 \%)$ in the non-EC group $(\mathrm{p}<0.003)$.

The ablation strategy after circumferential ablation was dependent on the operator. In the first 5 of 7 patients in the EC group in whom PVs were not isolated after circumferential ablation, repetitive electroanatomical mapping revealed the earliest potential at the carina region and we performed ablation at the carina resulting in PV isolation. In the last two patients in the EC group, the earliest activation area in the RA was mapped during pacing from the right inferior PV prior to carina ablation, as shown in Figure 6. The earliest area in the RA was broad, and extensive ablation to the area did not result in PV isolation. Eventually, focal ablation at the carina was required in both cases. In contrast, in 4 of the 29 patients (14\%) in the non-EC group in whom PV isolation was not achieved by circumferential ablation, subsequent electroanatomical mapping revealed an anterior gap in 2 patients $(7 \%)$, and ablation of that gap isolated the PVs. In the remaining 2 patients $(7 \%)$, subsequent electroanatomical mapping showed no gap in initial lesions and early activation at the carina of the RPV. Additive ablation at the carina was performed and the PVs were isolated.

\section{EAT and EC}

Periatrial and intercaval EAT volumes were significantly lower in the EC group than in the non-EC group $\left(12.8 \pm 6.2\right.$ vs. $23.1 \pm 13.9 \mathrm{ml} / \mathrm{m}^{2}, \mathrm{p}<0.02$, and $1.1 \pm 0.8$ vs. $2.2 \pm 1.6 \mathrm{ml} / \mathrm{m}^{2}, \mathrm{p}<0.02$, respectively; Figure 1C).

\section{Discussion}

\section{Major findings}

The main findings of the present study were as follows: (1) RAPW pacing revealed EC of the right PV in a considerable number of patients (29\%), while RAA pacing revealed no EC in any of the patients; (2) for achieving PV isolation, ablation on the carina was more frequently required in the EC group than in the non-EC group; and (3) patients in the EC group were younger and had less periatrial and intercaval EAT compared with those in the non-EC group.

\section{Pacing site, EC, and ablation outcome}

In the present study, all patients underwent LA electroanatomical mapping during both RAA and RAPW pacing. As shown in Figure 3, in the EC group, breakthroughs of the RPV carina and the LA anteroseptal region were detected only during RAPW pacing, although RAA pacing showed a breakthrough at the LA anteroseptal region only. Prior to the present study, Yoshida and colleagues identified 6 of the 34 (18\%) patients with right-sided carina breakthrough by electroanatomical mapping during sinus rhythm. ${ }^{6}$ Compared with their study, our study had a higher proportion of patients with right-sided carina breakthrough $(12 / 41,29 \%)$. These results are attributed to the fact that the site of impulse origin affects the choice of the propagation route. The sinus node is located in the anterior or anterolateral quarter of the superior vena cava and the RA junction, ${ }^{13}$ and the RAA is located anterior and medial to the RA. If the distance between the impulse origin and RAPW, where the intercaval bundle is assumed to be attached, is long, LA activation may be predominated by the Bachmann bundle at the LA anteroseptum in patients with right-sided carina 
breakthrough. Accordingly, the presence of EC may be underestimated by electroanatomical mapping during sinus rhythm or RAA pacing.

The majority of patients in the EC group required additive carina ablation to achieve PV isolation, compared to almost no patient in the non-EC group. However, two cases required carina ablation even in the non-EC group, suggesting that not only EC but also factors such as a thick PV carina can prevent PV isolation by circumferential ablation alone. Another possibility is that that EC could not be accurately detected in these cases. The exact location of the epicardial fiber attachment on the RAPW was difficult to determine; therefore, inappropriate RAPW pacing could not depict EC, and carina ablation was indispensable. To solve such a problem, Hasebe et al. performed a simple pacing maneuver in patients in whom the right pulmonary vein was not isolated by circumferential ablation, and clearly separated EC from the gap of circumferential lesion. ${ }^{14}$ Their method may provide an alternative solution in cases which ECs are present but difficult to depict before ablation.

Furthermore, even in cases with EC, PV isolation was achieved by circumferential ablation only. This is probably because the circumferential lesions were proximal enough to PV to achieve isolation by ablating near the LA insertion of the intercaval bundle.

\section{Relationship between EAT, aging, and EC}

Our study suggests two potentially important contributors to the absence of a right-sided EC of the PV. The accumulation of periatrial/intercaval EAT and advancing age were significantly associated with the absence of an EC. There are several reasons why these factors were involved. The EAT is a metabolically active organ that secretes adipocytokines such as adiponectin, tumor necrosis factor- $\alpha$, interleukin 6 , monocyte chemoattractant protein- $1,{ }^{15}$ and Activin A. ${ }^{9}$ As there are no fascial boundaries between the EAT and the myocardium, these adipokines may have local pro-fibrotic and pro-inflammatory effects that facilitate fibrosis on the adjacent atrial myocardium. Indeed, the secretome from human EAT has been shown to induce fibrosis of the atrium in an organo-culture model of rat atria. ${ }^{9}$

Furthermore, in an experimental animal study conducted by Mahajan et al., ${ }^{11}$ persistent obesity was associated with significant myocardial fatty infiltration, EAT accumulation, and interstitial fibrosis with subsequent slowing of atrial conduction velocity. Such direct fatty infiltration and fibrosis separating myocytes could directly result in the slowing of regional conduction in a manner similar to microfibrosis. ${ }^{16}$ The conduction of the intercaval bundle, which connects the RA to the right PV on the epicardial side, is assumed to be blocked by infiltration and the local effect of adjacent EAT. A clinical study conducted by Hanaki et al. demonstrated that shorter interatrial distance between the posterior wall of the RA and the right PVs was associated with the necessity for carina ablation. ${ }^{17}$ In their study, the amount of EAT was not measured, but the fact that the interatrial distance was short could be assumed to mean that the EAT was small. This result seems to support the present study, which showed that a small amount of EAT can affect the presence of ECs.

Aging has also been shown to change electrical properties. A clinical study demonstrated aging to be associated with increased atrial conduction time. ${ }^{18}$ A human study conducted by Spach and Dolber also demonstrated the age-related development of extensive collagenous connective tissue that separates small groups of fibers and subsequently decreases atrial conduction. ${ }^{19}$ Hence, the age-related development of atrial fibrosis is an important contributor to the slowing of epicardial atrial conduction in elderly patients.

\section{Prior studies}

Several studies have reported an EC between the RPV carina and RA in patients undergoing PV isolation. However, the definition of EC is not standardized, and its assessment varies; it is sometimes performed during sinus rhythm before ablation ${ }^{6}$ or alternatively during atrial/PV pacing after ablation. ${ }^{7,8}$ Determining the presence or absence of epicardial breakthrough after ablation can make it difficult to distinguish residual LA-PV conduction from RA-PV epicardial breakthrough. Acute edema and enhancement on T2-weighted magnetic resonance imaging performed immediately after AF ablation correlate significantly with low-voltage 
areas $(<0.05 \mathrm{mV})$ mapped using the CARTO system. ${ }^{20}$ Furthermore, acute post-ablation edema is observed not only in regions directly subjected to radiofrequency energy but also in distant regions. Consequently, even in the presence of residual conduction sites after circumferential ablation, early apparent potentials may not be recorded at or around the initial ablation line, and instead only at sites away from the initial lesion, such as the carina, which may mimic the distal attachment of EC. Electroanatomical mapping of LA breakthrough should be performed prior to ablation to avoid the influence of non-transmural ablation or edematous lesions. Two studies have described the disconnection of epicardial connection by ablation in the RA. In the present study, we attempted to ablate the RA end of epicardial fibres in two patients; however, cluster ablation of the RAPW did not achieve PV isolation. As indicated in Figure 6, the earliest area in the RA was extensive and may be difficult to ablate from the RA. Ablation of the carina may be essential for PV isolation.

\section{Clinical implications}

In this study, RAPW pacing could detect the EC that can interrupt PV isolation by antral circumferential ablation, and such an occurrence was higher in patients with a low EAT volume and in relatively younger patients. There has been a dramatic improvement in the efficacy of transmural ablation by using an irrigation catheter, contact force, and the ablation index/lesion size index. ${ }^{21}$ With these technologies, it is presumed that there are only a few cases in which PV isolation is not achieved because of the inability to achieve circumferential lesions transmurally, and EC may play a more important role in the resistance of PV isolation. Therefore, in relatively younger patients or patients with a small EAT volume, the presence of carina conductivity should be assessed before AF ablation, as early detection of carina conduction may help avoid unnecessary additional antral ablation and collateral damage.

\section{Limitations}

First, our sample size was small, which thus requires validation by large-scale studies to prove the findings of the present study. Second, a possibility of far-field capture of the RPV carina by RAPW pacing was observed that may have affected the main results of this study. However, the conduction time between the RAPW pacing stimulus and the RPV carina in the EC group was not short (48.5 $\pm 12 \mathrm{~ms})$ and far-field capture of the RPV carina is unlikely. Lastly, conduction regarding the ligament of Marshall and the left PVs was not evaluated in the present study, because assessment of the vein of Marshall is difficult. It cannot be determined whether age or epicardial fatty tissue is involved in this conduction.

\section{Conclusions}

In nearly a quarter of patients of our study, LA breakthrough via the carina was present, indicating EC between the RA and right PV carina. RAPW pacing can depict these ECs. To achieve PV isolation, carina ablation was required in a majority of the patients with EC. Large EAT volume and aging were associated with the absence of EC.

\section{Acknowledgements}

We thank Noboru Kitamura and Takeru Takada for their technical assistance during the electrophysiological study and the associated data acquisition.

\section{References}

1. Calkins H, Hindricks G, Cappato R, et al. 2017 HRS/EHRA/ECAS/APHRS/SOLAECE expert consensus statement on catheter and surgical ablation of atrial fibrillation. Europace 2018;20:e1-e160. 2. Kiuchi $\mathrm{K}$, Kircher S, Watanabe N, et al. Quantitative analysis of isolation area and rhythm outcome in patients with paroxysmal atrial fibrillation after circumferential pulmonary vein antrum isolation using the paceand-ablate technique. Circ Arrhythm Electrophysiol 2012;5:667-675. 3. Lin YJ, Tsao HM, Chang SL, et al. The distance between the vein and lesions predicts the requirement of carina ablation in circumferential pulmonary vein isolation. Europace 2011;13:376-382. 4. Ho SY, Cabrera JA, Tran VH, Farre J, Anderson RH, Sanchez-Quintana. Architecture of the pulmonary veins: relevance to radiofrequency ablation. Heart 
2001;86:265-270. 5. Ho SY, Cabrera JA, Sanchez-Quintana D. Left atrial anatomy revisited. Circ Arrhythm Electrophysiol 2012; 5:220-228. 6. Yoshida K, Baba M, Shinoda Y, et al. Epicardial connection between the right-sided pulmonary venous carina and the right atrium in patients with atrial fibrillation: a possible mechanism for preclusion of pulmonary vein isolation without carina ablation. Heart Rhythm 2019;16:671-678. 7. Barrio-Lopez MT, Sanchez-Quintana D, Garcia-Martinez J, et al. Epicardial connections involving pulmonary veins: the prevalence, predictors, and implications for ablation outcome. Circ Arrhythm Electrophysiol 2020;13:e007544. 8. Patel PJ, D'Souza B, Saha P, Chik WWB, Riley MP, Garcia FC. Electroanatomic mapping of the intercaval bundle in atrial fibrillation. Circ Arrhythm Electrophysiol 2014;7:1262-1267. 9. Venteclef N, Guglielmi V, Balse E, et al. Human epicardial adipose tissue induces fibrosis of the atrial myocardium through the secretion of adipo-fibrokines. Eur Heart J 2015;36:795-805. 10. Hatem SN, Sanders P. Epicardial adipose tissue and atrial fibrillation. Cardiovasc Res 2014;102:205-213. 11. Mahajan R, Lau DH, Brooks AG, et al. Electrophysiological, electroanatomical, and structural remodeling of the atria as consequences of sustained obesity. J Am Coll Cardiol 2015;66:1-11. 12. Okamatsu H, Koyama J, Sakai Y, et al. High-power application is associated with shorter procedure time and higher rate of first-pass pulmonary vein isolation in ablation index-guided atrial fibrillation ablation. J Cardiovasc Electrophysiol 2019;30:2751-2758. 13. Ho SY, Sánchez-Quintana D. Anatomy and pathology of the sinus node. J Interv Card Electrophysiol 2016;46:3-8. 14. Hasebe H, Yoshida K, Nogami A, et al. A simple pacing maneuver to unmask an epicardial connection involving the right-sided pulmonary veins. J Cardiovasc Electrophysiol. 2021;32:287-296. 15. Mazurek T, Zhang L, Zalewski A, et al. Human epicardial adipose tissue is a source of inflammatory mediators. Circulation 2003; 108:2460-2466. 16. Spach MS, Boineau JP. Microfibrosis produces electrical load variations due to loss of side-to-side cell connections: a major mechanism of structural heart disease arrhythmias. Pacing Clin Electrophysiol 1997;20:397-413. 17. Hanaki Y, Yoshida K, Baba M, et al. Interatrial distance predicts the necessity of additional carina ablation to isolate the right-sided pulmonary veins. Heart Rhythm O2. 2020;1:259-267. 18. Kistler PM, Sanders P, Fynn SP, et al. Electrophysiologic and electroanatomic changes in the human atrium associated with age. J Am Coll Cardiol 2004;44:109-116. 19. Spach MS, Dolber PC. Relating extracellular potentials and their derivatives to anisotropic propagation at a microscopic level in human cardiac muscle. Evidence for electrical uncoupling of side-to-side fiber connections with increasing age. Circ Res 1986;58:356-371. 20. Vergara GR, Marrouche NF. Tailored management of atrial fibrillation using a LGE-MRI based model: from the clinic to the electrophysiology laboratory. J Cardiovasc Electrophysiol 2011;22:481-487. 21. Ariyarathna N, Kumar S, Thomas SP, et al. Role of contact force sensing in catheter ablation of cardiac arrhythmias: evolution or history repeating itself? JACC Clin Electrophysiol 2018; 4:707-723. 
A
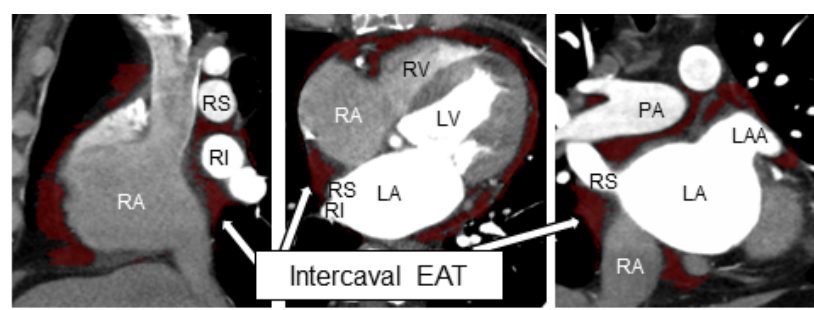
Volume
Atrial EAT $31.3 \mathrm{ml} / \mathrm{m}^{2}$

Intercaval EAT $2.5 \mathrm{ml} / \mathrm{m}^{2}$

B
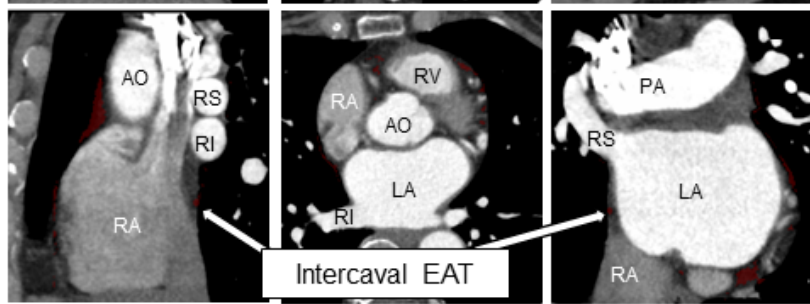

(1)
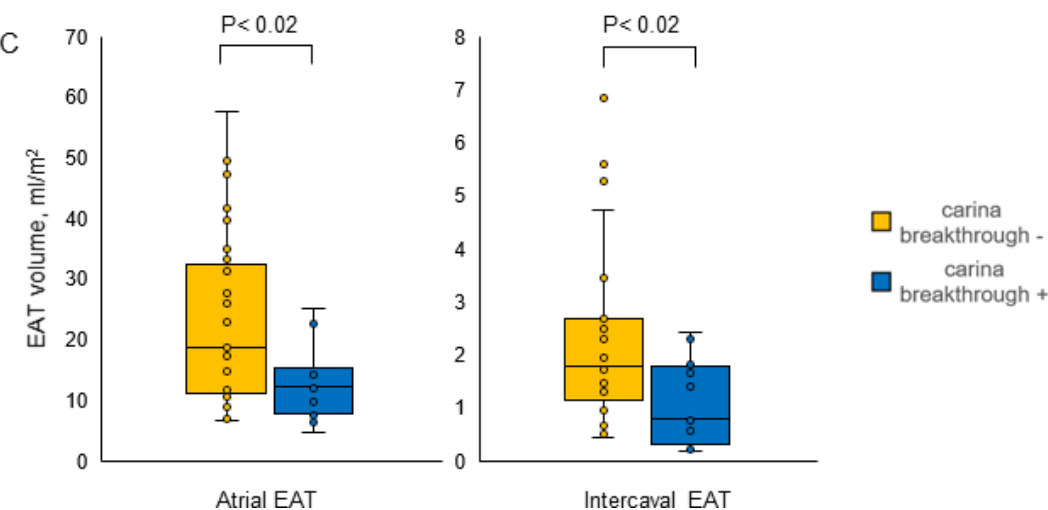

Figure 1. Differences in Epicardial Adipose Tissue Volume Among Patients without and with Epicardial Connection

Representative samples of computed tomography images of epicardial adipose tissue (EAT, indicated in dark red) in a patient (A) without epicardial connection and (B) with epicardial connection. The images are shown in the sagittal plane at the level of the intercaval area (left panel), in the axial plane at the level of the right pulmonary vein carina (mid panel), and in the coronal plane at the level of the right pulmonary vein carina (right panel). Note that in the upper image of patients without epicardial connection, the EAT volume in the intercaval area is larger (indicated by a white arrow in the upper panel) than that of patients with epicardial connection (indicated by a white arrow in the lower panel). The comparison of periatrial and intercaval EAT volumes between the two groups are shown in (C). EAT = epicardial adipose tissue; PA $=$ pulmonary artery; $\mathrm{RS}=$ right superior pulmonary vein; $\mathrm{RI}=$ right inferior pulmonary vein; Ao = aorta; $\mathrm{RA}=$ right atrium; $\mathrm{RV}=$ right ventricle; $\mathrm{LAA}=$ left atrial appendage. 


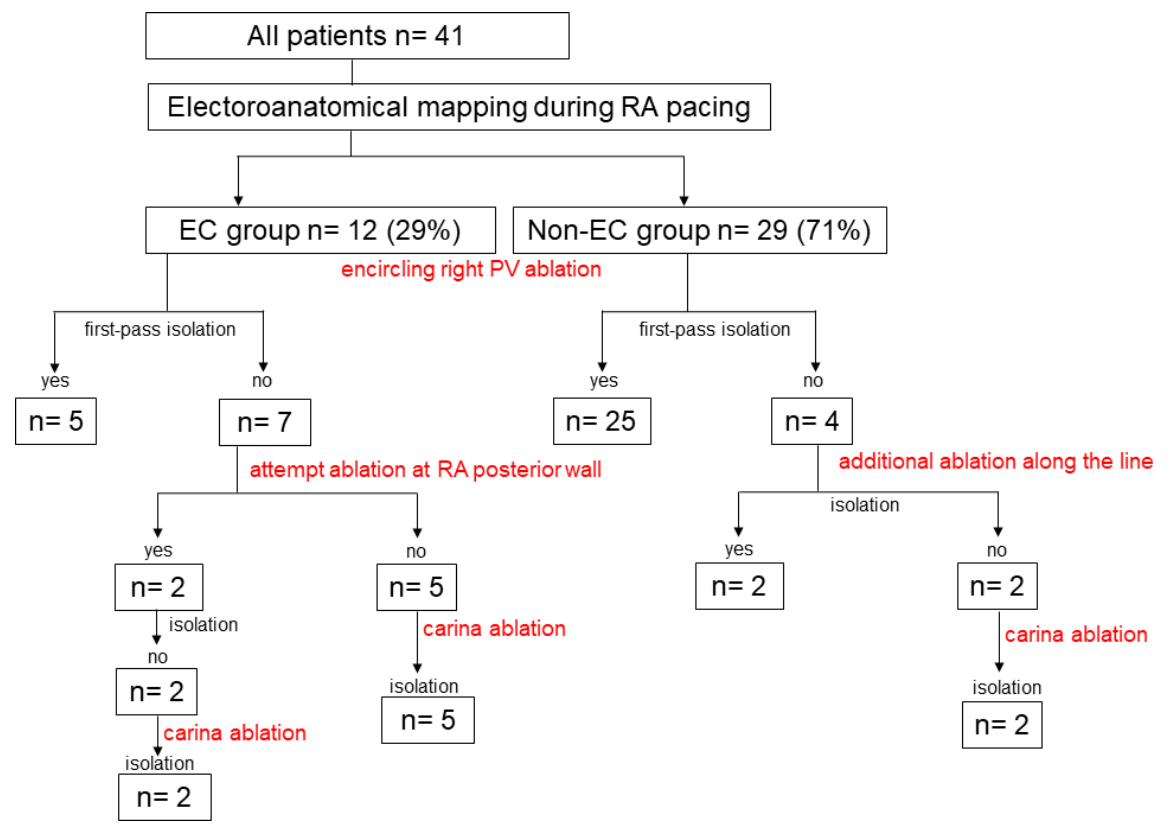

Figure 2. Study and Ablation Protocols

$\mathrm{EC}=$ epicardial connection; $\mathrm{PV}=$ pulmonary vein; $\mathrm{RA}=$ right atrium

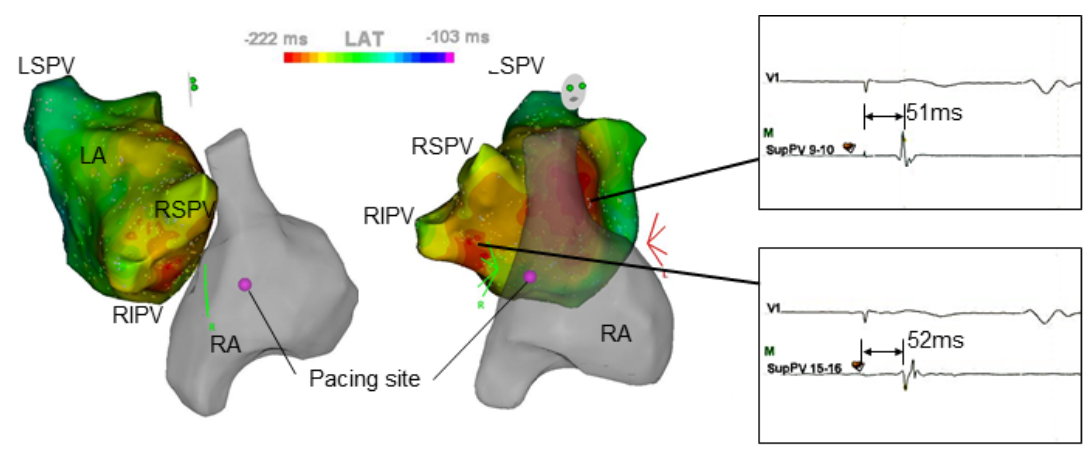

Pacing from the RA posterior wall

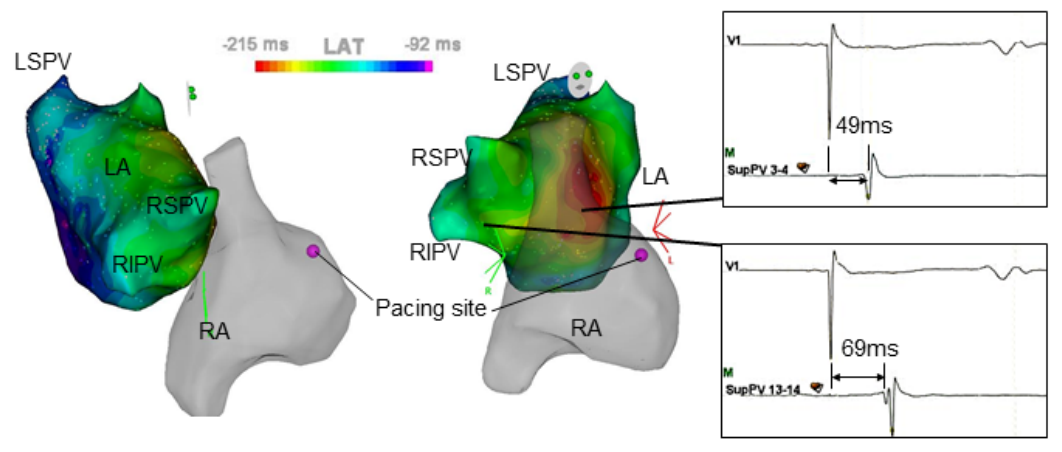

Pacing from the RA appendage 
Figure 3. Activation Maps Showing Left Atrial Breakthrough during Right Atrial Pacing in a Patient with Epicardial Connection

Representative atrial activation maps demonstrating left atrial breakthrough in a patient with epicardial connection. Local latency at the anteroseptal and carina regions are shown in each map. A magenta tag indicates the pacing site in the right atrium on each map. Activation map shows double breakthroughs at both the anteroseptal region and the carina. The epicardial connection is observed as one of the double breakthroughs and depicted only during right atrial posterior wall pacing.

$\mathrm{LA}=$ left atrium; RA = right atrium; LSPV = left superior pulmonary vein; LIPV = left inferior pulmonary vein; RSPV = right superior pulmonary vein; RIPV = right inferior pulmonary vein.

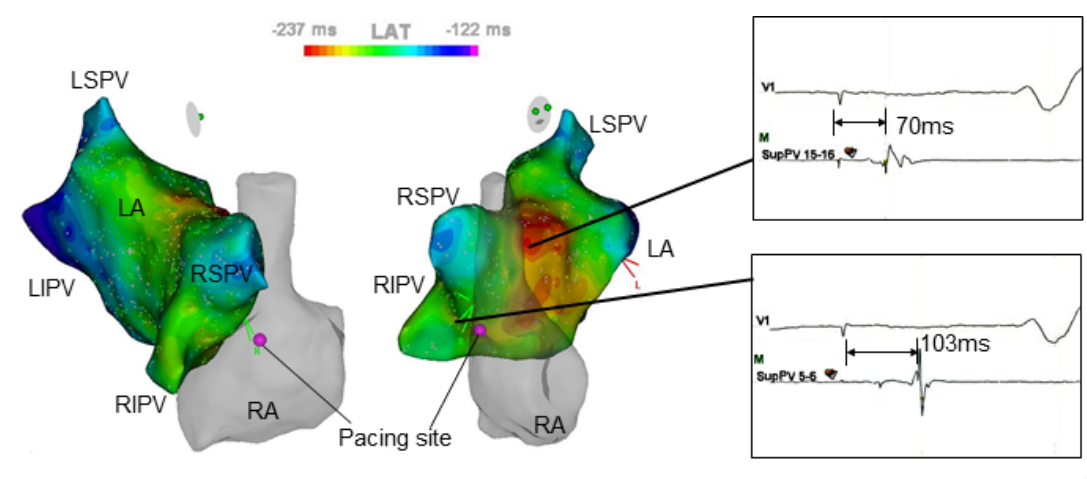

Pacing from the RA posterior wall

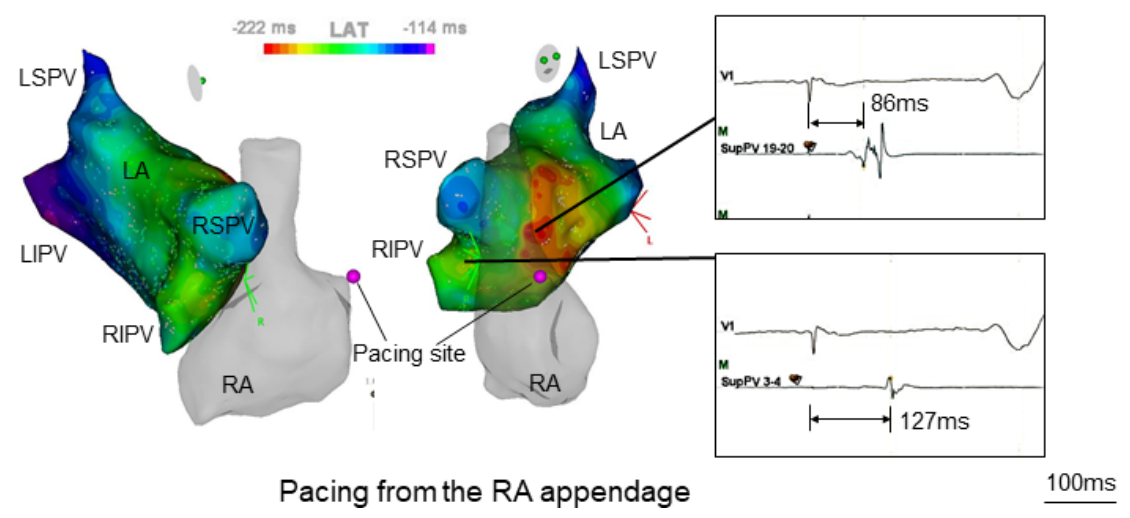

Figure 4 Activation Maps Showing Left Atrial Breakthrough during Right Atrial Pacing in a Patient without Epicardial Connection

Representative atrial activation maps demonstrating left atrial breakthrough in a patient with epicardial connection. Local latency at the anteroseptal and carina regions are shown in each map. A magenta tag indicates the pacing site in the right atrium on each map. The activation map shows a breakthrough only at the anteroseptal region. Left atrial activations were similar during right atrial appendage pacing and right atrial posterior wall pacing.

$\mathrm{LA}=$ left atrium; RA = right atrium; LSPV = left superior pulmonary vein; LIPV = left inferior pulmonary vein; RSPV = right superior pulmonary vein; RIPV = right inferior pulmonary vein. 


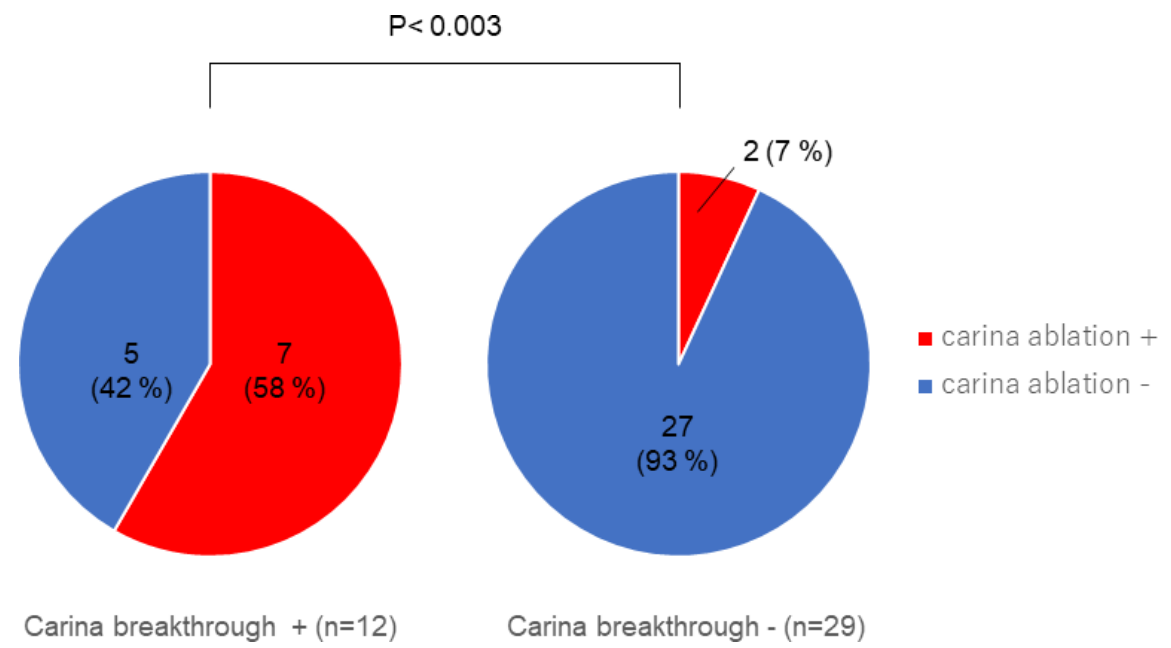

Figure 5. Proportions of Additive Carina Ablation for Achieving PV Isolation

The requirement for carina ablation in patients with and without carina breakthrough.

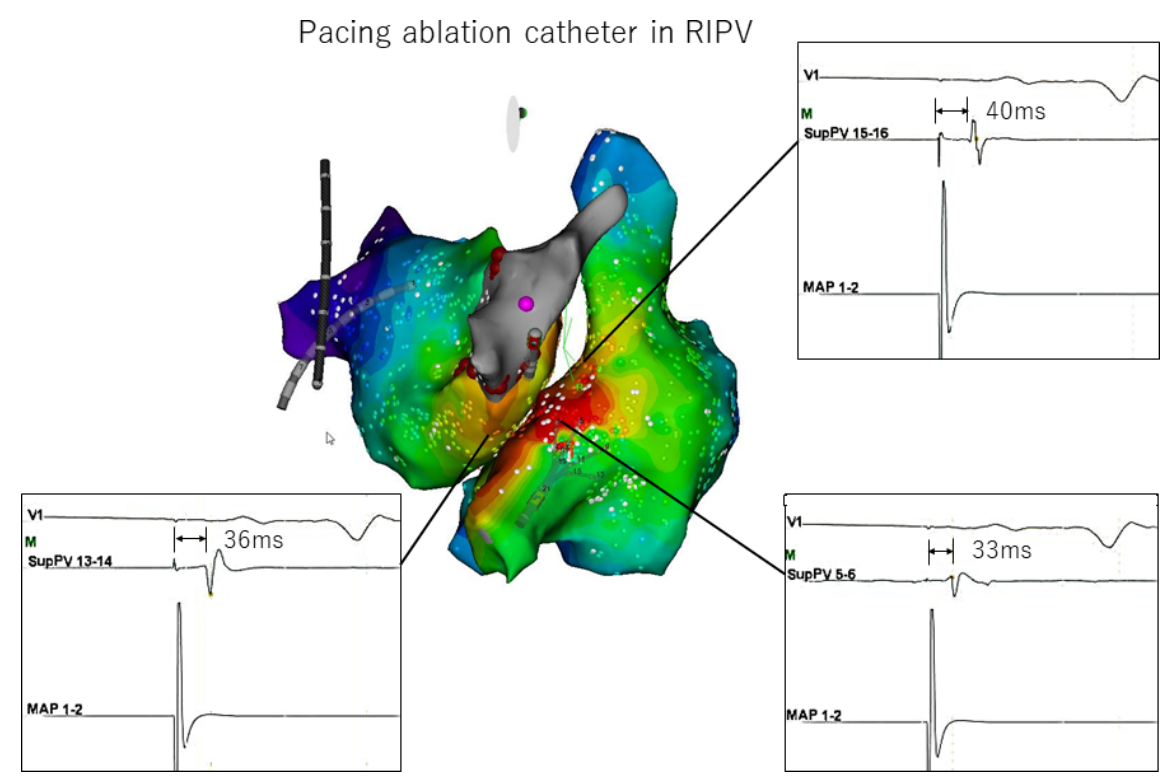

Figure 6. LA Activation Map during Pacing from the Right Inferior PV

The earliest site was broad and located in the RA posterior wall. Extensive ablation at the site did not result in PV isolation. Single radiofrequency application at the right PV carina (indicated by a pink tag) achieved isolation of the right PVs. LA, left atrium; PV, pulmonary vein; RA, right atrium; RIPV, right inferior PV.

\section{Tables}

Table 1. Patient characteristics

\begin{tabular}{llll}
\hline & EC group $(\mathbf{n}=\mathbf{1 2})$ & Non-EC group $(\mathbf{n}=\mathbf{2 9})$ & p-value \\
\hline Age, years & $66.5 \pm 6.6$ & $72.4 \pm 8.3$ & 0.021 \\
Sex (female), n & $5(41.7)$ & $12(41.4)$ & 1
\end{tabular}




\begin{tabular}{llll}
\hline & EC group $(\mathbf{n}=\mathbf{1 2})$ & Non-EC group $(\mathbf{n = 2 9 )}$ & p-value \\
\hline Persistent AF, n & $7(58.3)$ & $19(65.5)$ & 0.73 \\
LA diameter, mm & $39.9 \pm 5.0$ & $39.7 \pm 6.5$ & 0.94 \\
Hypertension, $\mathrm{n}$ & $5(41.7)$ & $17(58.6)$ & 0.493 \\
Diabetes mellitus, $\mathrm{n}$ & $2(16.7)$ & $7(24.1)$ & 0.47 \\
Congestive heart failure, $\mathrm{n}$ & $4(33.3)$ & $10(34.4)$ & 1 \\
Body mass index, $\mathrm{kg} / \mathrm{m}^{2}$ & $22.8 \pm 3.2$ & $24.3 \pm 3.9$ & 0.241 \\
LV ejection fraction, $\%$ & $53.1 \pm 14.8$ & $58.4 \pm 11.1$ & 0.219 \\
$\beta$-Blocker, n & $8(66.7)$ & $14(48.2)$ & 0.325 \\
Class Ia-c drug, n & $2(16.7)$ & $5(17.2)$ & 1 \\
Class III drug, n & $1(8.3) 11$ & $1(3.4)$ & 0.505 \\
Digoxin, n & $0(0)$ & $1(3.4)$ & 1 \\
\hline
\end{tabular}

Values are mean \pm standard deviation or $\mathrm{n}(\%)$

AF, atrial fibrillation; EC, epicardial connection; LA, left atrium; LV, left ventricle

Table 2. Electroanatomical mapping data

\begin{tabular}{llll}
\hline & EC group $(\mathrm{n}=12)$ & Non-EC group $(\mathrm{n}=29)$ & $\mathrm{p}$-value \\
\hline Conduction time from the RAPW stimulus to the LA, ms & $48.5 \pm 12.2$ & $55.6 \pm 15.4$ & 0.167 \\
LA activation time during RAPW pacing, ms & $98.3 \pm 20.2$ & $107.2 \pm 19.7$ & 0.197 \\
Length of right PV encircling lesion, cm & $11.7 \pm 1.3$ & $12.6 \pm 1.6$ & 0.096 \\
Number of mapping points, n & $1428 \pm 481$ & $1287 \pm 268$ & 0.863 \\
\hline
\end{tabular}

Values are mean \pm standard deviation or $\mathrm{n}(\%)$

EC, epicardial connection; LA, left atrium; PV, pulmonary vein; RAPW, right atrial posterior wall 